\title{
Automatic exposure compensation in intraoral digital radiography: effect on the gray values of dental tissues
}

\author{
Evelyn Rute Carneiro Maciel' , Eduarda Helena Leandro Nascimento ${ }^{2 *}$, Hugo Gaêta-Araujo ${ }^{3}$, Maria Luiza \\ dos Anjos Pontual ${ }^{1}$, Andrea dos Anjos Pontual ${ }^{1}$ and Flávia Maria Moraes Ramos-Perez ${ }^{1}$
}

\begin{abstract}
Background: This study aimed to investigate the effect of automatic exposure compensation (AEC) of intraoral radiographic systems on the gray values of dental tissues in images acquired with or without high-density material in the exposed region using different exposure times and kilovoltages. The influence of the distance of the high-density material was also investigated.
\end{abstract}

Methods: Radiographs from the molar region of two mandibles were obtained using the RVG 6100 and the Express systems, operating at 60 and $70 \mathrm{kV}$ and $0.06,0.10$, and $0.16 \mathrm{~s}$. Subsequently, a titanium implant was inserted in the premolar's socket and other images were acquired. Using the ImageJ software, two regions of interest were determined on the enamel, coronary dentine, root dentine, and pulp of the first and second molars to obtain their gray values.

Results: In the RVG 6100, the implant did not affect the gray values ( $p>0.05)$; the increase in $\mathrm{kV}$ decreased it in all tissues $(p<0.05)$, and the exposure time affected only the root dentine and pulp. In the Express, only enamel and coronary dentine values changed $(p<0.05)$, decreasing with the implant presence and/or with the increase in exposure factors. The distance of the implant did not affect the results $(p>0.05)$.

Conclusions: AEC's performance varies between the radiographic systems. Its effect on the gray values depends not only on the presence or absence of high-density material but also on the $\mathrm{kV}$ and exposure time used.

Keywords: Dental radiography, Dental materials, Digital radiography, Digital system, Periapical radiography

\section{Background}

Digital image acquisition systems are increasingly present in dentistry due to their advantages compared to conventional films, such as requiring less exposure time and presenting the possibility of image enhancement, which avoid repetition and consequently reduce the radiation dose to the patient [1-3].

\footnotetext{
*Correspondence: eduarda.hln@gmail.com

${ }^{2}$ Present Address: Division of Oral Radiology, Department of Dentistry, Odontomed Imagem - Medical and Dental Services, Av. Dois Rios, 632, Ibura, Recife, Pernambuco 51.230-000, Brazil

Full list of author information is available at the end of the article
}

In some systems, image enhancement is also performed automatically by the software, after image acquisition, but before it is displayed on the monitor, through an image pre-processing tool called Automatic Exposure Compensation (AEC) [4-6]. This tool increases image contrast, modifying pixel values non-linearly, producing high-contrast radiographs $[7,8]$. Also, AEC can modify the signal-to-noise ratio, presenting a lower value (i.e. darker image) compared to systems without AEC [7, 9]. Previous studies have shown that AEC can improve the accuracy of diagnosis of proximal caries in under- and overexposed images [4]. It was also stated previously that 
the effect of AEC is influenced by the presence of highdensity objects $[6,10]$.

Galvão et al. [6], in an in vitro study, investigated the influence of the presence of high-density material, as well as its size and exposure time, on the gray values of radiodensity liquids equivalent to dental tissues. The authors concluded that a high-density material present in the image can influence the AEC adjustment, regardless of exposure time and radiographic system. However, the gray values of dental tissues were tested employing radiodensity equivalent liquids separately (one dental tissue at a time on each radiograph), which does not reflect the clinical condition. In addition, apart from radiographic systems and exposure time, other parameters may be applied for image acquisition, such as tube current and kilovoltage $(\mathrm{kV})$, which are mainly responsible for the final image density and contrast, respectively. By applying different $\mathrm{kV}$ (i.e. different beam energy), AEC could act differently in adjusting the shades of gray, as $\mathrm{X}$-rays beam with different energy reaches the image receptor.

$\mathrm{AEC}$ is an autonomous tool, and thus the pre-processing of the image cannot be controlled by the operator. Also, little is known about the real clinical effect of AEC on the gray values of dental tissues when high-density objects are present in the radiographed area, and the effect of the exposure parameters. Therefore, it is essential to understand its functioning and how it can be influenced by factors present in the radiographed area in clinical situations (i.e., presence of high-density materials) and technical parameters (i.e. $\mathrm{kV}$ and exposure time).

The aim of the present study was to evaluate, in an $e x$ vivo model, the effect of AEC on dental tissues (enamel, crown dentine, root dentine, and pulp), using two different radiographic systems, under different acquisition parameters (exposure time and $\mathrm{kV}$ ). In addition, the potential influence of the distance from the high-density material on the gray values was tested. The null hypothesis was that the effect of AEC does not influence the gray values of dental tissues, regardless of the variables considered in the study.

\section{Methods}

This study was approved by the Research Ethics Committee of the Federal University of Pernambuco (protocol \#3.594.302). All procedures performed were under the ethical standards of the Institutional and National Research Committee and with the 1964 Helsinki Declaration and its later amendments or comparable ethical standards. The informed consent requirement for this ex vivo study was waived by the Research Ethics Committee of the Federal University of Pernambuco, and permission to use the ex vivo sample was granted by this committee.
The study sample consisted of periapical radiographs obtained from two partially edentulous human mandibles belonging to the Oral Radiology Department used for teaching purposes, selected considering the following inclusion criteria: mandibles containing adjacent first and second molars on at least one side, and absence of the second premolar. Molars with dental restoration or endodontic treatment and mandibles with moderate to advanced bone loss in the molar region were excluded. Finally, two molar regions (one in each mandible) were available for the acquisition of the study images.

\section{Image acquisition}

All images were acquired using the Heliodent Plus X-ray machine (Sirona Dental Systems, Bensheim, Germany) and two digital intraoral systems:

- A Complementary metal-oxide-semiconductor, direct radiography (DR) system, 12-bit depth-RVG 6100 (Kodak Dental Systems, New York, USA);

- A photostimulable phosphor (PSP) plate, computed radiography (CR) system, 14-bit depth-Express (Instrumentarium, Tuusula, Finland).

For both systems, the X-ray machine was set to operate at $7 \mathrm{~mA}$ (tube current not adjustable), two kilovoltages $(60$ and $70 \mathrm{kV})$, and three exposure times $(0.06,0.10$, and $0.16 \mathrm{~s})$. In each mandible, periapical radiographs of the right mandibular molars were acquired with and without the insertion of an $11 \times 4 \mathrm{~mm}$ titanium dental implant (Titamax, Neodent, Brazil) in the alveolus of the second premolar, composing two experimental groups: control group (without high-density material in the radiographed region) and high-density group (with the dental implant in the radiographed region, placed within the edentulous socket of the right second premolar). Table 1 summarizes the study's protocols and variables.

Images were acquired using the parallelism technique. An acrylic device was used to standardize the geometric relationships between the X-ray source/object/image receiver. Between the acquisitions of the control group and the high-density group, the mandible was not moved, so that the exact spatial relationship between it and the

Table 1 Exposure protocols and study variables

\begin{tabular}{llll}
\hline Radiographic system & Group & kV & Time (s) \\
\hline RVG 6100 & Control & 60 and 70 & $0.06-0.10-0.16$ \\
Express & High-density & 60 and 70 & $0.06-0.10-0.16$ \\
& Control & 60 and 70 & $0.06-0.10-0.16$ \\
& High-density & 60 and 70 & $0.06-0.10-0.16$ \\
\hline
\end{tabular}




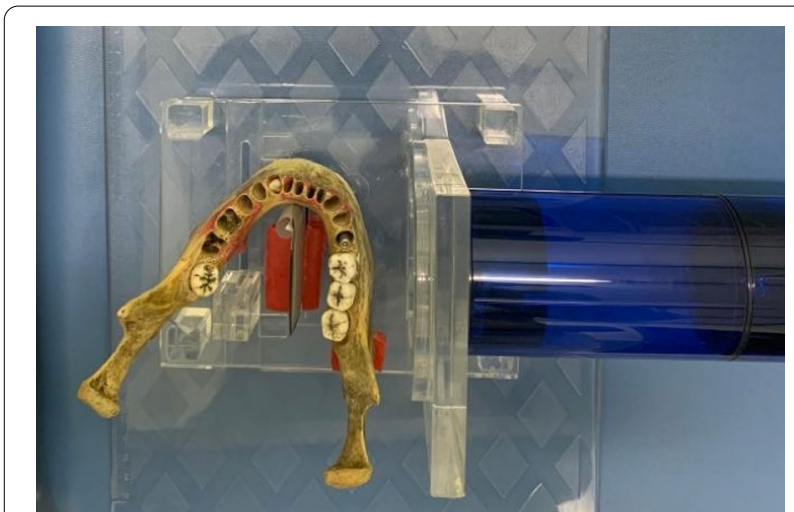

Fig. 1 Acquisition of the radiographic image with the Express system (high-density group)

image receiver was kept the same throughout the study (Fig. 1).

Considering the objective nature of the analyses proposed in the present study, each acquisition was repeated 5 times [6]. The repetitions of acquisitions also aimed to verify the reproducibility of the method and record the possible variability of gray values between acquisitions. Thus, 240 radiographs were acquired ( 2 molar regions $\times 2$ radiographic systems $\times 2$ groups $\times 2 \mathrm{kV} \times 3$ exposure times $\times 5$ repetitions).

\section{Objective assessment of gray values of dental tissues}

To have a standardization between the two systems used, all images were saved in TIFF format and exported with 8-bit depth to ImageJ software (U.S. National Institutes of Health, Bethesda, Maryland, USA). In the radiographs of each mandible, two regions of interest (ROIs) of size 0.65 $\mathrm{mm} \times 0.65 \mathrm{~mm}$ were determined on the enamel, crown dentine, root dentine, and pulp of the evaluated teeth (Fig. 2). The ROIs were recorded in the ImageJ software using the ROI manager tool, which standardizes the ROI position on all images. In each ROI, the mean and standard deviation (SD) of the gray values of each tissue were recorded.

\section{Statistical analysis}

The mean and SD values of each tissue were tabulated in an Excel spreadsheet (Microsoft Corporation, Redmond, WA, USA) and analyzed using the SPSS software (v. 22.0, IMB Corp., Armonk, New York, USA). Two-way analysis of variance (ANOVA) was used to compare the gray values between the groups tested (control and high-density) with the varying protocol ( $\mathrm{kV}$ and exposure time) within each tissue and radiographic system, followed by Tukey's post-hoc test for multiple comparisons. An additional analysis was performed to evaluate the influence of the

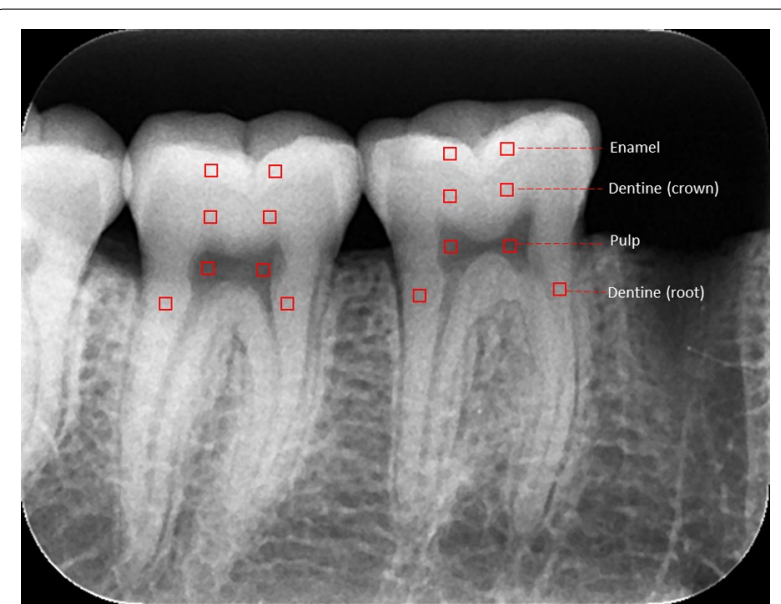

Fig. 2 Representation of the square regions of interest located in the enamel, dentin (crown), dentin (root), and pulp of the first and second molars in an image obtained with the Express system

distance to the high-density material on the mean gray values. For that, the difference between the gray values $(\Delta$ gray values) in the high-density group and control group were calculated for the ROIs placed on enamel and dentine (both crown and root) of the first molar and the second molar, and compared by t-test. Those comparisons were performed only for those protocols that showed a statistically significant difference in the first part of the study. The significance level was set at $5 \%(\alpha=0.05)$.

\section{Results}

The results related to the RVG 6100 system are shown in Table 2. For this system, the presence of the implant did not significantly affect the gray values of any of the dental tissues, regardless of the exposure time and the $\mathrm{kV}(p=0.895)$. However, there were differences between the images acquired with 60 and $70 \mathrm{kV}(p<0.001)$ : for all dental tissues, images obtained with higher $\mathrm{kV}$ showed lower gray values (i.e., darker image). By comparing the images obtained in the same $\mathrm{kV}$ with different exposure times, it was observed that the time affected only the gray values of the tissues of lower physical density (root dentine and pulp) $(p<0.001)$. In the control group, the increase from 0.06 to $0.16 \mathrm{~s}$ decreased the gray values of the root dentine at $70 \mathrm{kV}$ and of the pulp at both kilovoltage $(p=0.026)$; in the high-density group, images acquired with $0.16 \mathrm{~s}$ showed lower gray values than the others, when $70 \mathrm{kV}$ was used $(p<0.001)$.

Table 3 details the results for the Express system, which shows that only the gray values of enamel and crown dentine underwent significant changes according to the study variables $(p<0.001)$. The presence of the implant caused a decrease in the gray values of 
Table 2 Mean and standard deviation (SD) of the gray values of dental tissues in the RVG 6100 radiographic system distributed according to the group, exposure time, and kilovoltage

\begin{tabular}{|c|c|c|c|c|c|c|c|}
\hline \multirow[t]{2}{*}{ Dental tissues } & \multirow[t]{2}{*}{ Group } & \multicolumn{2}{|l|}{$t=0.06 \mathrm{~s}$} & \multicolumn{2}{|l|}{$\mathrm{t}=0.10 \mathrm{~s}$} & \multicolumn{2}{|l|}{$t=0.16 \mathrm{~s}$} \\
\hline & & $60 \mathrm{kV}$ & $70 \mathrm{kV}$ & $60 \mathrm{kV}$ & $70 \mathrm{kV}$ & $60 \mathrm{kV}$ & $70 \mathrm{kV}$ \\
\hline \multirow[t]{2}{*}{ Enamel } & Control & 135.0 (12.7) Aa & 118.1 (13.8) Ab & 139.2 (11.9) Аa & 118.2 (12.9) Ab & 141.7 (13.2) $\mathrm{Aa}$ & 115.4 (13.3) Ab \\
\hline & High-density & 134.8 (12.7) Aa & 118.4 (13.1) Ab & 139.6 (12.1) Aa & 119.2 (13.2) Ab & 142.2 (13.3) Aa & 116.1 (13.7) Ab \\
\hline \multirow[t]{2}{*}{ Crown dentine } & Control & 112.2 (11.8) Aa & 93.1 (11.4) Ab & 114.3 (10.5) Aa & 91.9 (10.3) Ab & 115.4 (11.3) Aa & 88.5 (10.5) Ab \\
\hline & High-density & 111.7 (11.5) Aa & 93.1 (10.7) Ab & 114.6 (10.5) Aa & 93.0 (10.8) Ab & 116.0 (11.4) Aa & 89.1 (10.9) Ab \\
\hline \multirow[t]{2}{*}{ Root dentine } & Control & $97.3(6.5) \mathrm{Aa}$ & 78.9 (6.3) Ab & $98.5(8.4) \mathrm{Aa}$ & 77.4 (7.8) Abc & $99.2(9.1) \mathrm{Aa}$ & 73.8 (7.9) Ac \\
\hline & High-density & $97.5(6.1) \mathrm{Aa}$ & $79.3 \mathrm{Ab}$ & 99.6 (7.7) $\mathrm{Aa}$ & 78.9 (7.1) Ab & 100.5 (8.0) Aa & 74.9 (6.8) Ab \\
\hline \multirow[t]{2}{*}{ Pulp } & Control & 49.7 (6.1) Aa & $35.2(5.1) \mathrm{Ac}$ & 47.2 (5.8) Aab & 32.6 (4.3) Acd & 46.2 (5.6) Ab & 29.8 (4.1) Ad \\
\hline & High-density & 48.9(6.2) $\mathrm{Aa}$ & $35.0(5.0) \mathrm{Ab}$ & $47.7(5.8) \mathrm{Aa}$ & 33.7 (4.7) Ab & $47.0(5.7) \mathrm{Aa}$ & $30.3(4.1) \mathrm{Ac}$ \\
\hline
\end{tabular}

Different uppercase letters indicate a statistical difference between the control and high-density groups, within each dental tissue (vertical comparisons) Different lowercase letters indicate a statistical difference between $\mathrm{kV}$ at the same exposure time and between exposure times at the same $\mathrm{kV}$, within each group (horizontal comparisons)

Table.3 Mean and standard deviation (SD) of the gray values of dental tissues in the Express radiographic system distributed according to the group, exposure time, and kilovoltage

\begin{tabular}{|c|c|c|c|c|c|c|c|}
\hline \multirow[t]{2}{*}{ Dental tissue } & \multirow[t]{2}{*}{ Group } & \multicolumn{2}{|l|}{$t=0.06 s$} & \multicolumn{2}{|l|}{$t=0.10 \mathrm{~s}$} & \multicolumn{2}{|l|}{$\mathrm{t}=0.16 \mathrm{~s}$} \\
\hline & & $60 \mathrm{kV}$ & $70 \mathrm{kV}$ & $60 \mathrm{kV}$ & $70 \mathrm{kV}$ & $60 \mathrm{kV}$ & $70 \mathrm{kV}$ \\
\hline \multirow[t]{2}{*}{ Enamel } & Control & $227.5(8.4) \mathrm{Aa}$ & 225.1 (10.3) Aab & 226.0 (9.1) Aab & 215.5 (10.1) AC & 220.5 (10.8) Ab & $213.1(10.8) A C$ \\
\hline & High-density & $224.2(10.4) \mathrm{Aa}$ & $217.2(9.6) \mathrm{BC}$ & 224.0 (10) Aab & 208.5 (10.7) Bd & 217.7 (11.5) Ab & 205.4 (8.3) Bd \\
\hline \multirow[t]{2}{*}{ Crown dentine } & Control & 192.4 (13.4) Aa & 190.5 (10.4) Aab & 193.2 (11.6) Aa & $181.0(11.1) \mathrm{AC}$ & $185.0(8.8) \mathrm{Abc}$ & 185.2 (16) Abc \\
\hline & High-density & 187.7 (12.3) Aa & 182.4 (9.7) Bab & 189.5 (11) Aa & $173.4(8.4) \mathrm{Bc}$ & 180.2 (9) Abc & 177.1 (11) Bbc \\
\hline \multirow[t]{2}{*}{ Root dentine } & Control & $172.4(28.8) \mathrm{Aa}$ & 169.6 (22.4) Aa & 171.9 (26.6) Aa & 166.5 (21.4) Aa & 167.7 (22.7) Aa & $171.6(26.4) \mathrm{Aa}$ \\
\hline & High-density & 168.5 (25.3) Aa & 162.7 (22.9) $\mathrm{Aa}$ & $167.7(25.2) \mathrm{Aa}$ & 158.4 (19.4) Aa & $162.3(21.5) \mathrm{Aa}$ & 164.1 (22.1) Aa \\
\hline \multirow[t]{2}{*}{ Pulp } & Control & 83.5 (24.3) Aa & $95.6(20.1) \mathrm{Aa}$ & 90.5 (24.5) Aa & 98.1 (17.6) $\mathrm{Aa}$ & 93.4 (20.8) Aa & 92.4 21.5) $\mathrm{Aa}$ \\
\hline & High-density & 77.4 (22.4) Aa & $89.4(20.8) \mathrm{Aa}$ & 82.5 (23.2) Aa & 93.6 (17.6) $\mathrm{Aa}$ & 88.9 (19.8) Aa & 88.3 (19.8) Aa \\
\hline
\end{tabular}

Different uppercase letters indicate a statistical difference between the control and high-density groups, within each dental tissue (vertical comparisons)

Different lowercase letters indicate a statistical difference between $\mathrm{kV}$ at the same exposure time and between exposure times at the same $\mathrm{kV}$, within each group (horizontal comparisons)

both dental tissues at $70 \mathrm{kV}$, regardless of the exposure time $(p<0.001)$. The increase in $\mathrm{kV}$ decreased the values of crown dentine at $0.10 \mathrm{~s}$ and enamel at 0.10 and $0.16 \mathrm{~s}$ in the control and high-density groups, and of enamel at $0.06 \mathrm{~s}$ in the high-density group $(p<0.001)$. The increase in the exposure time from 0.06 to $0.16 \mathrm{~s}$ decreased the gray values of the enamel at both $\mathrm{kV}(p=0.047)$ and of the crown dentine at $60 \mathrm{kV}$ $(p=0.036)$.

The influence of the distance to the high-density material was tested only for those variables (Express system and $\mathrm{kV}$ ) and dental tissues (enamel and crown dentine) that presented significant differences in the first evaluation. No difference was observed for the variation of the average gray values ( $\Delta$ Gray Values) (control imageshigh-density images) between the first molar and second molar, in any of the kV and dental tissues tested (Fig. 3; $p>0.08)$.

\section{Discussion}

It has been demonstrated here that the performance of the AEC depends not only on the presence or absence of high-density material in the radiographed area but also on the $\mathrm{kV}$ and exposure time used and the physical density of the radiographed anatomical structures. All these possible variables might influence on one system but not on the other, as the AEC can act differently for each digital system, as previously reported $[6,10]$.

In the RVG 6100 system, the presence of the implant did not significantly affect the gray values of the dental tissues in the exposure times and $\mathrm{kV}$ used. According to our knowledge, this was the first time that a system that present AEC did not show significant changes in any of the gray values of the structures present in the radiographed area when a high-density material was introduced into it, differently from what was found in other studies that also carried out similar evaluations $[6,10]$. 


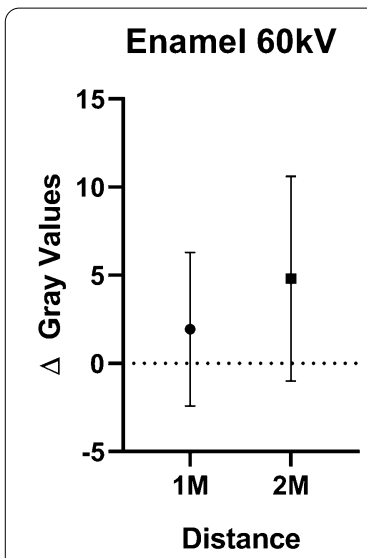

Enamel 70kV

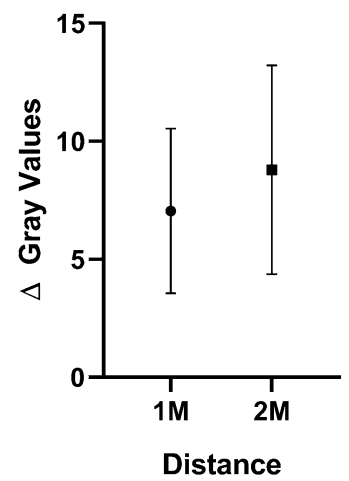

Fig. 3 Graphs showing the mean $\Delta$ gray values and standard deviation between high-density and control groups for enamel and dentine of the first (1 M) and second molars (2 M) using $60 \mathrm{kV}$ and $70 \mathrm{kV}$ with an exposure time of $0.06 \mathrm{~s}$ for the Express system

Such fact may be attributed to the fact that previous studies did not simulate a clinical scenario for this evaluation. The clinical disposition of the dental tissues and the attenuation of X-rays by bone and soft tissues covering (this latter represented here by an acrylic plate) may be factors that reduce the final effect of AEC activation by a high-density object.

In the Express system, only the gray values of enamel and crown dentine change significantly, decreasing with the presence of the implant (at $70 \mathrm{kV}$ ) and/or with the increase in exposure factors. This result differs from the study by Galvão et al. [6] in which all materials equivalent to dental tissues (pulp, enamel, and dentine) were influenced by the presence of a high-density material (e.g., implant). In the present study, we classified dentin as "crown" and "root" because, although dentin has the same physical density throughout the entire tooth, its radiographic density could change as a result of overlapping enamel or alveolar bone depending on the region evaluated.
Previous studies that evaluated the effect of AEC in the presence of high-density material obtained varying results for each digital system. Galvão et al. [6] evaluated this effect in three different systems at $70 \mathrm{kV}$ and demonstrated that, in two of them, Digora Toto (CMOS) and VistaScan (PSP), the presence of high-density material decreased the gray values of all equivalent dental tissue materials, regardless of the exposure time; while in the third system, Digora Optime (PSP), there was a decrease only in the pulp equivalent tissue and there was an increase in dentine equivalent tissue for 0.1 and $0.16 \mathrm{~s}$ and an increase in enamel equivalent tissue in $0.16 \mathrm{~s}$. Dashpuntsag et al. [10] also evaluated the Digora Optime system, at $60 \mathrm{kV}$, which demonstrated a similar operation to the Megadixel system (CCD) in the radiographs of a thin aluminum phantom: the addition of a high-density plate reduced the gray values and the contrast of the image. In the thick aluminum phantom, however, they presented opposite reactions: the gray values decreased in the Megadixel and increased in the Digora Optime system [10]. Therefore, it is noticed that the AEC is intensified by the presence of high-density material in the radiographed area can present different effects according to the digital system used. In addition, some of these systems, as in the case of Digora Optime, can have a bidirectional function, causing both increasing and decreasing gray values according to the exposed structure. It is worth emphasizing that these previous studies used phantoms with tissue-equivalent liquids representing each dental tissue individually [6] or aluminum scales [10] in their study designs, unlike ours that used real teeth and mandibles.

This was also the first study that evaluated the effect of the distance of the high-density material on the gray values of the adjacent dental tissues. This was achieved through a statistical evaluation of the difference of variations in gray values that occurred between the tissues of the first and second molars, with the introduction of the dental implant in the image. Thus, we observed that there was no significant influence of distance on any tissue. Differently, Galvão et al. [6] evaluated the size of the equivalent high-density material in the radiographed region, representing what would be a different amount of high-density material in the image, but also found a similar result: this factor did not affect the gray values significantly. Even so, as these authors did not use dental tissues for radiographic acquisition, the variable "amount of high-density material" in the radiographed area still needs to be further studied.

Regarding kilovoltage, in the RVG 6100 system, the increase in $\mathrm{kV}$ from 60 to 70 decreased the gray values in all dental tissues and exposure times, thus being the parameter that most caused changes in the image. In the 
Express system, the increase in $\mathrm{kV}$ decreased the gray values only for crown dentine and enamel (tissues of greater physical density). In theory, the increase in $\mathrm{kV}$ qualitatively modifies the X-ray photons, increasing their capability to pass through the tissues. In systems without the AEC, this also results, although indirectly, in the darkening of the radiograph (reduction of gray values), since more X-ray photons will reach the image receiver [5]. As this is expected when the AEC function is not present but also occurred in systems with AEC, it seems that the AEC does not interfere so strongly in the changes caused by $\mathrm{kV}$ in the image. Hence, it is shown the importance of taking $\mathrm{kV}$ into account when comparing studies and when translating these findings to clinical application. No previous study has demonstrated the influence of $\mathrm{kV}$ on AEC function, and this makes it difficult to compare our results.

The behavior of the radiographic systems studied was the opposite when we analyzed the results regarding the exposure time. In the RVG 6100 system, the increase in time decreased the gray values of pulp and root dentine, while in the Express system, the increase in exposure time decreased the values of enamel and crown dentine in some protocols. As is known, increased exposure usually causes the darkening of the entire radiograph. Thus, we could attribute to the AEC the fact that the tissues with higher and lower physical density were not affected in the RVG 6100 and Express, respectively. Dashpuntsag et al. [10] also evaluated the effect of the exposure time variation on radiographs from thin and thick aluminum scales. On the thin scale, the increase in time hardly caused changes in gray values in the Digora Optime system, while causing an important variation in Megadixel system. On the thick scale, increasing the time decreased the gray values in Digora and increased in Megadixel. This indicates that the effect of the variation in exposure time in AEC systems depends on the thickness of the exposed structures and the radiographic system used.

In the present study, the images were evaluated on an 8-bit scale instead of the original scale of each system to obtain a standardization between them and to compare our findings with previous studies, which have also used that methodology. This scale presents a more homogeneous distribution of gray values over the 254 possibilities between black and white, while larger scales may group the values in a more heterogeneous way (which makes it more difficult to identify statistical differences in the variation of gray values). As for the use of the ImageJ software, it is a software that is widely used in radiological studies with methodologies similar to ours [6]. This software, in addition to precisely measuring the gray values in the chosen scale, also enabled us to standardize the size and location of the ROIs in the images, through its
ROI Manager tool. Such an exact standardization would hardly be achieved in the software of each device.

The results obtained here are not sufficient to fully explain the action of the AEC in the gray values of the tissues, given that the AEC is an automatic tool whose performance is not disclosed by the manufacturers. According to Hayawaka et al. [8], the AEC function algorithm produces a high-contrast image irrespective of exposure, in a non-linear manner. Thus, the AEC algorithm could provide compensation for different degrees of exposure by stretching the range of pixel values to increase the image contrast, probably detecting the minimum and maximum pixel values of the image, redistributing and shifting these values to change the displayed image [8]. As AEC activity cannot be turned off by the operator, it is not possible to compare with the results of the same system without AEC. Our findings, however, clarify that the effect of AEC in a clinical situation differs from that in assessments of the radiographic density of liquids or aluminum scales $[6,10]$.

In addition, few studies have evaluated AEC from a clinical perspective, such as the effect of AEC on the accuracy of diagnosing proximal caries [4] and the repercussion of the presence of high-density material on this diagnostic task [11], which presented controversial results. Dashpuntsag et al. [10] also demonstrated that the alteration of contrast in the image caused by AEC can impair the accuracy of other diagnostic tasks, such as the detection of minimal bone regeneration in periodontal treatments, root cracks, and fractures. Likewise, the change of gray values by AEC can seriously affect the results of radiographic density surveys, especially those in which a clinical scenario is not simulated, such as in vitro studies of the density of types of cement and resins and other dental materials. Thus, the presence of AEC in radiographic systems can represent disadvantages in its use. Moreover, the X-ray unit used in the present study had a fixed tube current at $7 \mathrm{~mA}$. The tube current, as the exposure time, is directly related to the number of photons produced and reaching the receptor. Therefore, one may expect that tube current and exposure time could impact the AEC intensity similarly. Future studies evaluating the effect of AEC on gray values of dental tissues in clinical conditions varying exposure parameters in other digital systems are encouraged. In addition, investigating AEC influence on other diagnostic tasks is also recommended.

\section{Conclusions}

In conclusion, AEC's performance varies between digital radiographic systems. Its effect on the gray values of dental tissues depends not only on the presence or absence 
of a high-density material in the radiographed area but also on the $\mathrm{kV}$ and exposure time used.

\section{Abbreviations}

AEC: Automatic exposure compensation; kV: Kilovoltage; ROl: Region of interest; SD: Standard deviation.

\section{Acknowledgements}

Not applicable.

\section{Authors' contributions}

ERCM, EHLN, MLAP, and AAP acquired the images, EHLN, HGA and FMMRP analyzed and interpreted the images data regarding the gray values of dental tissues. All authors contributed to the writing of the manuscript. All authors read and approved the final manuscript.

\section{Funding}

This study was financed in part by the National Research Council-Brazil (CNPq).

\section{Availability of data and materials}

The datasets generated and analyzed during the current study are available from the corresponding author on reasonable request.

\section{Declarations}

\section{Ethics approval and consent to participate}

This study was approved by the Research Ethics Committee of the Federal University of Pernambuco (protocol \#3.594.302). All procedures performed were in accordance with the ethical standards of the Institutional and National Research Committee and with the 1964 Helsinki declaration and its later amendments or comparable ethical standards. The informed consent requirement for this ex vivo study was waived by the Research Ethics Committee of the Federal University of Pernambuco.

\section{Consent for publication}

Not applicable.

\section{Competing interests}

The authors declare that they have no competing interests.

\section{Author details}

${ }^{1}$ Department of Clinical and Preventive Dentistry, Federal University of Pernambuco (UFPE), Av. Prof. Artur de Sá, 329-481, Recife, Pernambuco 52171-011, Brazil. 2Present Address: Division of Oral Radiology, Department of Dentistry, Odontomed Imagem - Medical and Dental Services, Av. Dois Rios, 632, Ibura, Recife, Pernambuco 51.230-000, Brazil. ${ }^{3}$ Oral Radiology Area, School of Dentistry, Federal University of Alfenas (UNIFAL-MG), R. Gabriel Monteiro da Silva, 700, Alfenas, Minas Gerais 37130-001, Brazil.

Received: 12 September 2021 Accepted: 28 December 2021

Published online: 05 January 2022

\section{References}

1. Rovaris K, Vasconcelos K, dedo Nascimento EHL, Oliveira ML, Freitas DQ, Haiter-Neto F. Brazilian young dental practitioners' use and acceptance of digital radiographic examinations. Imaging Sci Dent. 2016;46:239-44.

2. Vandenberghe $B$, Jacobs $\mathrm{R}$, Bosmans $H$. Modern dental imaging: a review of the current technology and clinical applications in dental practice. Eur Radiol. 2010;20:2637-55.

3. van der Stelt PF. Better imaging. J Am Dent Assoc. 2008;139(SUPPL.):S7-13.

4. Yoshiura K, Nakayama E, Shimizu M, Goto TK, Chikui T, Kawazu T, et al. Effects of the automatic exposure compensation on the proximal caries diagnosis. Dentomaxillofac Radiol. 2005;34:140-4.

5. Stuart C, White MJP. Oral radiology. Principles and interpretation. 7th ed. Mosby/Elsevier; 2013.
6. Galvão NS, Nascimento EHL, Lima CAS, Freitas DQ, Haiter-Neto F, Oliveira ML. Can a high-density dental material affect the automatic exposure compensation of digital radiographic images? Dentomaxillofac Radiol. 2019;48:2-7.

7. Hayakawa Y, Farman AG, Scarfe WC, Kuroyanagi K. Technical report. Processing to achieve high-contrast images with computed dental radiography. Dentomaxillofac Radiol. 1996:25:211-4.

8. Hayakawa Y, Farman AG, Scarfe WC, Kuroyanagi K. Pixel value modification using RVG-4 automatic exposure compensation for instant highcontrast images. Oral Radiol. 1996;12:11-8.

9. Yoshiura K, Welander U, McDavid W, Li G, Shi X-Q, Kawazu T, et al. Perceptibility curves for the Digora system. Dentomaxillofac Radiol. 2003;32:191-7.

10. Dashpuntsag O, Yoshida M, Kasai R, Maeda N, Hosoki H, Honda E. Numerical evaluation of image contrast for thicker and thinner objects among current intraoral digital imaging systems. Biomed Res Int. 2017;2017:1-10.

11. Galvão NS, Leandro Nascimento EH, Gaêta-Araujo H, Freitas DQ, HaiterNeto F, Oliveira ML. Automatic exposure compensation and subjective image enhancement in the radiographic diagnosis of caries. Braz Oral Res. 2020:34:1-8.

\section{Publisher's Note}

Springer Nature remains neutral with regard to jurisdictional claims in published maps and institutional affiliations.
Ready to submit your research? Choose BMC and benefit from

- fast, convenient online submission

- thorough peer review by experienced researchers in your field

- rapid publication on acceptance

- support for research data, including large and complex data types

- gold Open Access which fosters wider collaboration and increased citations

- maximum visibility for your research: over 100M website views per year

At BMC, research is always in progress.

Learn more biomedcentral.com/submissions 